\title{
A Fixed Point Theorem for Quasi - Contractive Mappings on Cone Metric Space with Banach Algebras without Assumption of Normality
}

\author{
Eriola Sila*, Elida Hoxha, Silvana Liftaj \\ University of Tirana, Faculty of Natural Science, Department of Mathematics, Tirana, Albania. \\ * Corresponding author. Tel.: 00355693736535; email: erjola.liftaj@fshn.edu.al \\ Manuscript submitted December 28, 2018; accepted April 15, 2019. \\ doi: 10.17706/ijapm.2019.9.3.127-134
}

\begin{abstract}
In 2013, Liu and Xu introduced the concept of cone metric spaces over Banach algebras, replacing Banach spaces by Banach algebras. They proved some fixed point theorems in these spaces. Xu and Radenovic gave another proof for the results of Liu and Xu where the cone didn't have the normality.

In this paper we prove a new fixed point theorem for quasi - contractive mappings in cone metric space over Banach algebras. As an application of the main result, we give an example. Also the map in this example satisfies the conditions of our theorem but not the conditions of theorems of Liu and $\mathrm{Xu}$ and $\mathrm{Xu}$ amd Radenovic and it has a fixed point.
\end{abstract}

Key words: Banach algebra, cone metric space, fixed point, quasi - contractive mapping.

\section{Introduction}

Fixed point theory is one of the most importance subject of Functional Analysis. It is applicated in different fields as in Geometry, Differential Equations, Informatics, Physics. S. Banach [1] proved a fixed point theorem for contractive mappings in metric space. Then, there are many authors who have generalized this result, as Ciric [2], Rhoades [3]. Metric spaces are generalized by many authors to other spaces cone metric spaces. The concept of cone metric space was reviewed by authors Huang and Zhang [4] by replacing the real axes in the definition of the distance by an ordered Banach space. They proved the Banach theorem in cone metric space. Many authors have worked over these spaces, as Karapinar [5], Rezapour. Sh, Hamlbarani. R [6]. Later, in 2013, Liu and Xu [7] introduced the concept of cone metric spaces over Banach algebras, replacing Banach spaces by Banach algebras. They proved some fixed point theorems of generalized Lipschitz mappings where cone is normal. Then, in 2014, Xu and Radenovic [8] gave original proofs for the results of Liu and Xu [7], but now without the assumption of normality of cone.

In his paper, Rhoades [3] has collected various contractive mappings in metric space. One of them is quasi - contractive mapping

$$
T: X \rightarrow X, d(T x, T y) \leq \max \left\{d(x, y), d(x, T x), d(y, T y), \frac{d(x, T y)+d(y, T x)}{2}\right\}, \forall x, y \in X
$$

In this paper we present quasi contractive mappings in cone metric spaces over and we give a new fixed point result for quasi - contractive mappings in these spaces.

\subsection{Preliminaries}


Definition 1.1.1 [9] Let A be a real Banach space. In A, an operation of multiplication defined, that satifiesthe following properties (for all $x, y, z \in \mathrm{A}, \alpha \in R$ ):

1) $(x y) z=x(y z)$;

2) $(x+y) z=x z+y z$ and $x(y+z)=x y+x z$

3) $\alpha(x y)=(\alpha x) y=x(\alpha y)$;

4) $\|x y\| \leq\|x\|\|y\|$;

In this paper we assume that a Banach algebra has a unit element $e$ such that $e x=x e=x$ for all $x \in$ A. $x \in \mathrm{A}$ is said to be invertible if there is an inverse element $y \in \mathrm{A}$ such that $x y=y x=e$. The inverse of $x$ is denoted $x^{-1}$.

Proposition 1.1.1 ([10]) Let A be a Banach algebra with a unit $e$ and $x \in$ A. If the spectral radius $\rho(x)<1$, i.e., $\rho(x)=\lim _{n \rightarrow \infty}\|x\|^{\frac{1}{n}}=\inf _{n \geq 1}\|x\|^{\frac{1}{n}}<1$ then $e-x$ is invertible. Actually, $(e-x)^{-1}=$ $\sum_{i=0}^{\infty} x^{i}$

Let $P$ be a non-empty closed convex of a Banach algebra A.

$P$ is called cone $[1]$ if

1) $\{\theta, e\} \subset P$;

2) for every $x, y \in P$, and $\alpha, \beta \in R, \alpha x+\beta y \in P$;

3) for every $x, y \in P, x y \in P$;

4) if $x \in P$ and $-x \in P$ then $x=\theta$

where $\theta$ is the null of the Banach algebra A. For a given cone $P \subset \mathrm{A}$, we can define a partial ordering $\preccurlyeq$ with respect to $P$, by $x \leqslant y$ if and only if $y-x \in P$, and $x<\prec y$ if and only if $y-x \in$ int $P$, where int $P$ denotes the interior of $P . P$ is called a solid cone if int $P \neq \emptyset$. $P$ is called normal if there exists a positive constant $M>0$ such that for all $x, y \in \mathrm{A}, \theta \leqslant x \leqslant y \Rightarrow\|x\| \leq M\|y\|$.

Definition 1.1.2 [1] Let $X$ be a non-empty set and A be a real Banach algebra. Suppose that the mapping $d: X \times X \rightarrow$ A satisfies:

1) $\theta \leqslant d(x, y)$ for all $x, y \in X$ and $d(x, y)=\theta$ if and only if $x=y$;

2) $d(x, y)=d(y, x)$ for all $x, y \in X$;

3) $d(x, y) \leqslant d(x, z)+d(z, y)$ for all $x, y, z \in X$.

Thend is called a cone metric on $X$ and $(X, d)$ is called a cone metric space over the Banach algebra A.

Definition 1.1.3 [1] Let $(X, d)$ be a cone metric space over the Banach algebra A, $x \in X$ and let $\left\{x_{n}\right\}$ be a sequence in $X$. Then:

1) $\left\{x_{n}\right\}$ converges to $x$ if for each $c \in$ A with $\theta \ll c$, there exist a natural number $n_{0}$ such that for all $n>n_{0}, d\left(x_{n}, x\right) \ll c$. This is denoted by $\lim _{n \rightarrow \infty} x_{n}=x$ or $x_{n} \rightarrow x$.

2) $\left\{x_{n}\right\}$ is a Cauchy sequence if for each $c \in A$ with $\theta \ll c$, there exist a natural number $n_{0}$ such that for all $n, m>n_{0}, d\left(x_{n}, x_{m}\right) \ll c$.

3) $(X, d)$ is a complete cone metric space if every Cauchy sequence is convergent.

Definition 1.1.4 [5] Let $P$ a solid cone in a Banach space A. A sequence $\left\{x_{n}\right\}$ in $P$ is a $c$-sequence if for each $c \in$ A with $\theta \ll c$, there exist a natural number $n_{0}$ such that for all $n>n_{0}, x_{n} \ll c$.

Proposition 1.1.2 [8] Let $(X, d)$ be a complete cone metric space over the Banach algebra $\mathrm{A}$ and $P$ a solid cone in a Banach space A. If $\left\{x_{n}\right\}$ converges to $x$, then we have:

1) $\left\{d\left(x_{n}, x\right)\right\}$ is a $c$ - sequence.

2) $\left\{d\left(x_{n}, x_{n+p}\right)\right\}$ is a $c$ - sequence, for any $p \in N$.

Lemma 1.1.1 [8] Let $\mathrm{A}$ be a real Banach algebra and let $x, y \in \mathrm{A}$. If $x$ and $y$ commute, then the following hold:

1) $\rho(x, y) \leq \rho(x) \rho(y)$;

2) $\rho(x+y) \leq \rho(x)+\rho(y)$; 
3) $|\rho(x)-\rho(y)| \leq \rho(x-y)$.

Lemma 1.1.2 [8] Let A be a real Banach algebra and let $k \in$ A. If $0 \leq \rho(k)<1$, then $\rho\left((e-k)^{-1}\right) \leq$ $(1-\rho(k))^{-1}$.

Remark 1.1.1 [8] In general the condition $\rho(k)<1$ is weaker than $\|k\|<1$, and if $\rho(k)<1$ then $\left\|k^{n}\right\| \underset{n \rightarrow \infty}{\longrightarrow} 0$.

\section{Main Results}

Definition 2.1 Let $(X, d)$ be cone metric space over a Banach algebra A. The mapping $T: X \rightarrow X$ is called quasi contractive in $X$ if it satisfies:

$$
d(T x, T y) \preccurlyeq \operatorname{kmax}\{d(x, y), d(T x, x), d(T y, y), h[d(T x, y)+d(x, T y)]
$$

for every $x, y \in X$ and $k, h \in \mathrm{A}, \rho(k)<1$ and $\rho(h)<1$.

Theorem 2.1 Let $(X, d)$ be a complete cone metric space over a Banach algebra A, $P$ be a solid cone in A. Suppose that the mapping $T: X \rightarrow X$,is quasi - contractive, so it satisfies:

$$
d(T x, T y) \preccurlyeq k \max \{d(x, y), d(T x, x), d(T y, y), h[d(T x, y)+d(x, T y)]\}
$$

Where $h k=k h, \rho(k)<1$ and $\rho(h)<1$. Then $T$ has a fixed point in $X$.

Proof. Let $x_{0} \in X$, we construct the sequence $\left\{x_{n}\right\}_{n \in N}$ by $x_{n}=T x_{n-1}=T^{n} x_{0}, n \geq 1$.

We have $d\left(x_{n+1}, x_{n}\right)=d\left(T x_{n}, T x_{n-1}\right)$

$$
\begin{gathered}
\preccurlyeq k \max \left\{d\left(x_{n}, x_{n-1}\right), d\left(T x_{n}, x_{n}\right), d\left(T x_{n-1}, x_{n-1}\right), h\left[d\left(T x_{n}, x_{n-1}\right)+d\left(x_{n}, T x_{n-1}\right)\right]\right\} \\
=k \max \left\{d\left(x_{n}, x_{n-1}\right), d\left(x_{n+1}, x_{n}\right), d\left(x_{n}, x_{n-1}\right), h\left[d\left(x_{n+1}, x_{n-1}\right)+d\left(x_{n}, x_{n}\right)\right]\right\} \\
=k \max \left\{d\left(x_{n}, x_{n-1}\right), d\left(x_{n+1}, x_{n}\right), h d\left(x_{n+1}, x_{n-1}\right)\right\}
\end{gathered}
$$

Case 1. If $\max \left\{d\left(x_{n}, x_{n-1}\right), d\left(x_{n+1}, x_{n}\right), h d\left(x_{n+1}, x_{n-1}\right)\right\}=d\left(x_{n}, x_{n-1}\right)$, we have $d\left(x_{n+1}, x_{n}\right) \preccurlyeq k d\left(x_{n}, x_{n-1}\right) \leqslant k^{2} d\left(x_{n-1}, x_{n-2}\right) \leqslant \cdots \leqslant k^{n} d\left(x_{1}, x_{0}\right)$

Using the same technique as [8], we prove that the sequence $\left\{x_{n}\right\}_{n \in N}$ is Cauchy. Taking $n<m$, we have

$$
\begin{gathered}
d\left(x_{n}, x_{m}\right) \preccurlyeq d\left(x_{n}, x_{n+1}\right)+d\left(x_{n+1}, x_{n+2}\right)+\cdots+d\left(x_{m-1}, x_{m}\right) \\
\preccurlyeq\left(k^{n}+k^{n+1}+\cdots+k^{m-1}\right) d\left(x_{1}, x_{0}\right) \leq k^{n}\left(e+k+\cdots+k^{m-n-1}\right) d\left(x_{1}, x_{0}\right) \\
\preccurlyeq k^{n}\left(\sum_{i=0}^{\infty} k^{i}\right) d\left(x_{1}, x_{0}\right)=k^{n}(e-k)^{-1} d\left(x_{1}, x_{0}\right)
\end{gathered}
$$

Since $\rho(k)<1$ then $\left\|k^{n}\right\| \underset{n \rightarrow \infty}{\longrightarrow}$, so we have that $\left\|k^{n}(e-k)^{-1} d\left(x_{1}, x_{0}\right)\right\| \underset{n \rightarrow \infty}{\longrightarrow}$ 0. From Proposition 1.1.2 [8], for every $c \in \mathrm{A}$, with $c \gg 0$, there exist $n_{0} \in N$, such that for every $m>n>n_{0}$, we have:

$d\left(x_{n}, x_{m}\right) \leqslant k^{n}(e-k)^{-1} d\left(x_{1}, x_{0}\right) \ll c$. So the sequence $\left\{x_{n}\right\}_{n \in N}$ is Cauchy. Since $(X, d)$ is complete, the sequence $\left\{x_{n}\right\}_{n \in N}=\left\{T^{n} x_{0}\right\}_{n \in N}$ is convergent.

Case 2. If $\max \left\{d\left(x_{n}, x_{n-1}\right), d\left(x_{n+1}, x_{n}\right), h d\left(x_{n+1}, x_{n-1}\right)\right\}=d\left(x_{n+1}, x_{n}\right) \quad$, we have $d\left(x_{n+1}, x_{n}\right) \leqslant k d\left(x_{n+1}, x_{n}\right)$, so $(e-k) d\left(x_{n+1}, x_{n}\right) \leqslant \theta$. By multiplying by $(e-k)^{-1}$ both sides, we take $d\left(x_{n+1}, x_{n}\right) \leqslant \theta$, so $x_{n+1}=x_{n}=\cdots=x_{0}$. The sequence $\left\{x_{n}\right\}_{n \in N}$ is constant so it is convergent.

Case 3. If $\max \left\{d\left(x_{n}, x_{n-1}\right), d\left(x_{n+1}, x_{n}\right), h d\left(x_{n+1}, x_{n-1}\right)\right\}=h d\left(x_{n+1}, x_{n-1}\right)$, we have $d\left(x_{n+1}, x_{n}\right) \preccurlyeq$ $k h d\left(x_{n+1}, x_{n-1}\right) \leqslant k h\left[d\left(x_{n+1}, x_{n}\right)+d\left(x_{n}, x_{n-1}\right)\right], \quad$ and $\quad(e-k h) d\left(x_{n+1}, x_{n}\right) \leqslant k h d\left(x_{n}, x_{n-1}\right)$, so $d\left(x_{n+1}, x_{n}\right) \preccurlyeq(e-k h)^{-1} k h d\left(x_{n}, x_{n-1}\right)$. 
Write down $(e-k h)^{-1} k h=\lambda$, and we have $d\left(x_{n+1}, x_{n}\right) \preccurlyeq \lambda d\left(x_{n}, x_{n-1}\right) \preccurlyeq \lambda^{2} d\left(x_{n-1}, x_{n-2}\right) \leqslant \cdots \leqslant$ $\lambda^{n} d\left(x_{1}, x_{0}\right)$.

Now we have to prove that $\rho(\lambda)=\rho\left((e-k h)^{-1} k h\right)<1$.

Since $\rho(k)<1$ and $\rho(h)<1$ and $h k=k h$, we have $\rho(k h) \leq \rho(k) \rho(h)<1$. Noting that $(e-k h)^{-1}$ and $k h$ commute, $\rho\left((e-k h)^{-1} k h\right) \leq \rho\left((e-k h)^{-1}\right) \rho(k h) \leq(1-\rho(k h))^{-1} \rho(k h)<1$.

Now we prove that the sequence $\left\{x_{n}\right\}_{n \in N}$ is Cauchy. Taking $n<m$, we have

$$
\begin{gathered}
d\left(x_{n}, x_{m}\right) \preccurlyeq d\left(x_{n}, x_{n+1}\right)+d\left(x_{n+1}, x_{n+2}\right)+\cdots+d\left(x_{m-1}, x_{m}\right) \\
\preccurlyeq\left(\lambda^{n}+\lambda^{n+1}+\cdots+\lambda^{m-1}\right) d\left(x_{1}, x_{0}\right) \preccurlyeq \lambda^{n}\left(e+\lambda+\cdots+\lambda^{m-n-1}\right) d\left(x_{1}, x_{0}\right) \\
\preccurlyeq \lambda^{n}\left(\sum_{i=0}^{\infty} \lambda^{i}\right) d\left(x_{1}, x_{0}\right)=\lambda^{n}(e-\lambda)^{-1} d\left(x_{1}, x_{0}\right)
\end{gathered}
$$

Since $\rho(\lambda)<1$ then $\left\|\lambda^{n}\right\| \underset{n \rightarrow \infty}{\longrightarrow} 0$, so we have that $\left\|\lambda^{n}(e-\lambda)^{-1} d\left(x_{1}, x_{0}\right)\right\| \underset{n \rightarrow \infty}{\longrightarrow} 0$. For every $c \in A$, with $c \gg 0$, there exist $n_{0} \in N$, such that for every $m>n>n_{0}$, we have:

$$
d\left(x_{n}, x_{m}\right) \preccurlyeq \lambda^{n}(e-\lambda)^{-1} d\left(x_{1}, x_{0}\right) \ll c .
$$

So the sequence $\left\{x_{n}\right\}_{n \in N}$ is Cauchy. Since $(X, d)$ is complete, the sequence $\left\{x_{n}\right\}_{n \in N}=\left\{T^{n} x_{0}\right\}_{n \in N}$ is convergent.

In all cases, we proved that the sequence $\left\{x_{n}\right\}_{n \in N}$ is convergent. So, suppose that it converges to $x^{*}$.

Now we prove that $x^{*}$ is a fixed point of $T$, so $T x^{*}=x^{*}$.

$$
\begin{gathered}
d\left(T x^{*}, x^{*}\right) \leqslant d\left(T x^{*}, T x_{n}\right)+d\left(T x_{n}, x^{*}\right) \\
\leqslant k \max \left\{d\left(x^{*}, x_{n}\right), d\left(T x^{*}, x^{*}\right), d\left(T x_{n}, x_{n}\right), h\left[d\left(T x^{*}, x_{n}\right)+d\left(T x_{n}, x^{*}\right)\right]\right\}+d\left(T x_{n}, x^{*}\right)
\end{gathered}
$$

Case 1. If $\max \left\{d\left(x^{*}, x_{n}\right), d\left(T x^{*}, x^{*}\right), d\left(T x_{n}, x_{n}\right), h\left[d\left(T x^{*}, x_{n}\right)+d\left(T x_{n}, x^{*}\right)\right]\right\}=d\left(x^{*}, x_{n}\right)$ so we have

$$
d\left(T x^{*}, x^{*}\right) \preccurlyeq k d\left(x^{*}, x_{n}\right)+d\left(T x_{n}, x^{*}\right)
$$

Now, we see that the sequence $\left\{k d\left(x^{*}, x_{n}\right)+d\left(T x_{n}, x^{*}\right)\right\}$ is $c$ - sequence, because $\| k d\left(x^{*}, x_{n}\right)+$ $d\left(T x_{n}, x^{*}\right)\|\leq\| k\|\| d\left(x^{*}, x_{n}\right)\|+\| d\left(T x_{n}, x^{*}\right) \| \underset{n \rightarrow \infty}{\longrightarrow} 0$. For any $c \gg 0, d\left(T x^{*}, x^{*}\right) \leqslant k d\left(x^{*}, x_{n}\right)+d\left(T x_{n}, x^{*}\right) \ll$ $c$, so $d\left(T x^{*}, x^{*}\right)=0$ and $T x^{*}=x^{*}$.

Case 2. If $\max \left\{d\left(x^{*}, x_{n}\right), d\left(T x^{*}, x^{*}\right), d\left(T x_{n}, x_{n}\right), h\left[d\left(T x^{*}, x_{n}\right)+d\left(T x_{n}, x^{*}\right)\right]\right\}=d\left(T x^{*}, x^{*}\right)$ we have $d\left(T x^{*}, x^{*}\right) \leqslant k d\left(T x^{*}, x^{*}\right)+d\left(T x_{n}, x^{*}\right)$ and $(e-k) d\left(T x^{*}, x^{*}\right) \leqslant d\left(T x_{n}, x^{*}\right)$.

So $d\left(T x^{*}, x^{*}\right) \preccurlyeq(e-k)^{-1} d\left(T x_{n}, x^{*}\right)$. The sequence $\left\{(e-k)^{-1} d\left(T x_{n}, x^{*}\right)\right\}$ is $c-$ sequence, because $\left\|(e-k)^{-1} d\left(T x_{n}, x^{*}\right)\right\| \underset{n \rightarrow \infty}{\longrightarrow} 0$. For any $c \gg 0, d\left(T x^{*}, x^{*}\right) \preccurlyeq(e-k)^{-1} d\left(T x_{n}, x^{*}\right) \ll c$, so $d\left(T x^{*}, x^{*}\right)=0$ and $T x^{*}=x^{*}$.

Case 3. If $\max \left\{d\left(x^{*}, x_{n}\right), d\left(T x^{*}, x^{*}\right), d\left(T x_{n}, x_{n}\right), h\left[d\left(T x^{*}, x_{n}\right)+d\left(T x_{n}, x^{*}\right)\right]\right\}=d\left(T x_{n}, x_{n}\right)$ we have $d\left(T x^{*}, x^{*}\right) \leqslant k d\left(T x_{n}, x_{n}\right)+d\left(T x_{n}, x^{*}\right) \leqslant k d\left(T x_{n}, x^{*}\right)+k d\left(x^{*}, x_{n}\right)+d\left(T x_{n}, x^{*}\right)$.

The sequence $\left\{k d\left(T x_{n}, x^{*}\right)+k d\left(x^{*}, x_{n}\right)+d\left(T x_{n}, x^{*}\right)\right\}$ is $c$ - sequence, because $\| k d\left(T x_{n}, x^{*}\right)+$ $k d\left(x^{*}, x_{n}\right)+d\left(T x_{n}, x^{*}\right) \| \underset{n \rightarrow \infty}{\longrightarrow} 0$. For any $c \gg 0, d\left(T x^{*}, x^{*}\right) \preccurlyeq k d\left(T x_{n}, x^{*}\right)+k d\left(x^{*}, x_{n}\right)+d\left(T x_{n}, x^{*}\right) \ll c$, so $d\left(T x^{*}, x^{*}\right)=0$ and $T x^{*}=x^{*}$.

Case 4. If $\max \left\{d\left(x^{*}, x_{n}\right), d\left(T x^{*}, x^{*}\right), d\left(T x_{n}, x_{n}\right), h\left[d\left(T x^{*}, x_{n}\right)+d\left(T x_{n}, x^{*}\right)\right]\right\}=h\left[d\left(T x^{*}, x_{n}\right)+d\left(T x_{n}, x^{*}\right)\right]$, we have $d\left(T x^{*}, x^{*}\right) \preccurlyeq k h\left[d\left(T x^{*}, x_{n}\right)+d\left(T x_{n}, x^{*}\right)\right]+d\left(T x_{n}, x^{*}\right) k \preccurlyeq h\left[d\left(T x^{*}, x^{*}\right)+d\left(x^{*}, x_{n}\right)+d\left(T x_{n}, x^{*}\right)\right]+$ 
$d\left(T x_{n}, x^{*}\right)$. So $(e-k h) d\left(T x^{*}, x^{*}\right) \preccurlyeq k h d\left(x^{*}, x_{n}\right)+(e+k h) d\left(T x_{n}, x^{*}\right)$.

Furthermore, since $\rho(h k)<1$, we have

$$
d\left(T x^{*}, x^{*}\right) \preccurlyeq(e-k h)^{-1}\left[k h d\left(x^{*}, x_{n}\right)+(e+k h) d\left(T x_{n}, x^{*}\right)\right]
$$

Reasoning as in the other cases we get: for any $c \gg 0, d\left(T x^{*}, x^{*}\right) \ll c$, so $d\left(T x^{*}, x^{*}\right)=0$ and $T x^{*}=x^{*}$. So $x^{*}$ is a fixed point of $T$.

Example 2.1. Let $\mathrm{A}=R^{2}$. For each $x=\left(x_{1}, x_{2}\right) \in \mathrm{A}$, let $\|x\|=\left|x_{1}\right|+\left|x_{2}\right|$. The multiplication is defined by $x y=\left(x_{1}, y_{1}\right)\left(x_{2}, y_{2}\right)=\left(x_{1} y_{1}, x_{2} y_{2}\right)$. The unit of Banach algebra $\mathrm{A}$ is $e=(1,1)$.

Let $P=\left\{\left(x_{1}, x_{2}\right) \in R^{2} \mid x_{1} \geq 0, x_{2} \geq 0\right\}$ and $X=[0,1]$. A cone metric over Banach algebra is defined by $d(x, y)=\left\{\begin{array}{ll}(\max (x, y), \max (x, y)) & x \neq y \\ (0,0) & x=y\end{array}\right.$.

$(X, d)$ is a complete cone metric space over the Banach algebra $\mathrm{A}$.

Now define mapping $T: X \rightarrow X$ by $T x=\frac{1}{4} x^{2}+\frac{1}{2} x$ and $k=\left(\frac{7}{8}, \frac{7}{8}\right), h=\left(\frac{2}{3}, \frac{2}{3}\right), \rho\left(\left(\frac{7}{8}, \frac{7}{8}\right)\right)<1$ and $\rho\left(\left(\frac{2}{3}, \frac{2}{3}\right)\right)<1$

$$
d(T x, T y)=\left(\max \left(\frac{1}{4} x^{2}+\frac{1}{2} x, \frac{1}{4} y^{2}+\frac{1}{2} y\right), \max \left(\frac{1}{4} x^{2}+\frac{1}{2} x, \frac{1}{4} y^{2}+\frac{1}{2} y\right)\right)
$$

Suppose $x<y$.

Case 1. If $x<\frac{1}{4} y^{2}+\frac{1}{2} y<y$, we have $d(T x, T y)=\left(\frac{1}{4} y^{2}+\frac{1}{2} y, \frac{1}{4} y^{2}+\frac{1}{2} y\right)$

Now we see

$$
\begin{gathered}
d(x, y)=(\max (x, y), \max (x, y))=(y, y), \\
d(T x, x)=\left(\max \left(x, \frac{1}{4} x^{2}+\frac{1}{2} x\right), \max \left(x, \frac{1}{4} x^{2}+\frac{1}{2} x\right)\right)=(x, x), \\
d(T y, y)=\left(\max \left(y, \frac{1}{4} y^{2}+\frac{1}{2} y\right), \max \left(y, \frac{1}{4} y^{2}+\frac{1}{2} y\right)\right)=(y, y), \\
d(T x, y)=\left(\max \left(\frac{1}{4} x^{2}+\frac{1}{2} x, y\right), \max \left(\frac{1}{4} x^{2}+\frac{1}{2} x, y\right)\right)=(y, y), \\
d(T y, x)=\left(\max \left(\frac{1}{4} y^{2}+\frac{1}{2} y, x\right), \max \left(\frac{1}{4} y^{2}+\frac{1}{2} y, x\right)\right)=\left(\frac{1}{4} y^{2}+\frac{1}{2} y, \frac{1}{4} y^{2}+\frac{1}{2} y\right), \\
d(T x, y)+d(T y, x)=\left(\frac{1}{4} y^{2}+\frac{3}{2} y, \frac{1}{4} y^{2}+\frac{3}{2} y\right), \\
h[d(T x, y)+d(T y, x)]=\left(\frac{2}{3}, \frac{2}{3}\right)\left(\frac{1}{4} y^{2}+\frac{3}{2} y, \frac{1}{4} y^{2}+\frac{3}{2} y\right)=\left(\frac{1}{6} y^{2}+y, \frac{1}{6} y^{2}+y\right) .
\end{gathered}
$$

So

$$
\begin{gathered}
\max \{d(x, y), d(T x, x), d(T y, y), h[d(T x, y)+d(x, T y)]\}=\max \left\{(x, x),(y, y),\left(\frac{1}{6} y^{2}+y, \frac{1}{6} y^{2}+y\right)\right\} \\
=\left(\frac{1}{6} y^{2}+y, \frac{1}{6} y^{2}+y\right) .
\end{gathered}
$$

Now we have:

$k \max \{d(x, y), d(T x, x), d(T y, y), h[d(T x, y)+d(x, T y)]\}=\left(\frac{7}{8}, \frac{7}{8}\right)\left(\frac{1}{6} y^{2}+y, \frac{1}{6} y^{2}+y\right)=\left(\frac{7}{48} y^{2}+\frac{7}{8} y, \frac{7}{48} y^{2}+\right.$ $\left.\frac{7}{8} y\right)$. 
It's clearly that for each $y \in[0,1], \frac{7}{48} y^{2}+\frac{7}{8} y>\frac{1}{4} y^{2}+\frac{1}{2} y$, so

$$
d(T x, T y) \preccurlyeq k \max \{d(x, y), d(T x, x), d(T y, y), h[d(T x, y)+d(x, T y)]\}
$$

Case 2. If $\frac{1}{4} y^{2}+\frac{1}{2} y<x<y$, we have $d(T x, T y)=\left(\frac{1}{4} y^{2}+\frac{1}{2} y, \frac{1}{4} y^{2}+\frac{1}{2} y\right)$

Now we see

$$
\begin{gathered}
d(x, y)=(\max (x, y), \max (x, y))=(y, y), \\
d(T x, x)=\left(\max \left(x, \frac{1}{4} x^{2}+\frac{1}{2} x\right), \max \left(x, \frac{1}{4} x^{2}+\frac{1}{2} x\right)\right)=(x, x), \\
d(T y, y)=\left(\max \left(y, \frac{1}{4} y^{2}+\frac{1}{2} y\right), \max \left(y, \frac{1}{4} y^{2}+\frac{1}{2} y\right)\right)=(y, y), \\
d(T x, y)=\left(\max \left(\frac{1}{4} x^{2}+\frac{1}{2} x, y\right), \max \left(\frac{1}{4} x^{2}+\frac{1}{2} x, y\right)\right)=(y, y), \\
d(T y, x)=\left(\max \left(\frac{1}{4} y^{2}+\frac{1}{2} y, x\right), \max \left(\frac{1}{4} y^{2}+\frac{1}{2} y, x\right)\right)=(x, x) . \\
d(T x, y)+d(T y, x)=(x+y, x+y), \\
h[d(T x, y)+d(T y, x)]=\left(\frac{2}{3}, \frac{2}{3}\right)(x+y, x+y)=\left(\frac{2}{3}(x+y), \frac{2}{3}(x+y)\right) .
\end{gathered}
$$

So

$$
\begin{gathered}
\max \{d(x, y), d(T x, x), d(T y, y), h[d(T x, y)+d(x, T y)]\}= \\
\max \left\{(x, x),(y, y),\left(\frac{2}{3}(x+y), \frac{2}{3}(x+y)\right)\right\}=(y, y) .
\end{gathered}
$$

Now we have $k \max \{d(x, y), d(T x, x), d(T y, y), h[d(T x, y)+d(x, T y)]\}=\left(\frac{7}{8}, \frac{7}{8}\right)(y, y)=\left(\frac{7}{8} y, \frac{7}{8} y\right)$. It's clearly that for each $y \in[0,1], \frac{7}{8} y>\frac{1}{4} y^{2}+\frac{1}{2} y$, so

$$
d(T x, T y) \preccurlyeq k \max \{d(x, y), d(T x, x), d(T y, y), h[d(T x, y)+d(x, T y)]\}
$$

Thus $T$ is quasi contractive in $X$, and we are in the condition of Theorem 2.1, so $T$ has a fixed point $x=0$.

Remark 2.1. We see that this example doesn't complete the conditions of Theorem 3.2 [8], because it doesn't exist any $k$ such that $\rho(k)<\frac{1}{2}$ and $d(T x, T y) \leqslant k[d(T x, y)+d(T y, x)]$ for all $x, y \in X$.

So if we take $x<\frac{1}{4} y^{2}+\frac{1}{2} y<y$,

$$
d(T x, T y)=\left(\frac{1}{4} y^{2}+\frac{1}{2} y, \frac{1}{4} y^{2}+\frac{1}{2} y\right) \preccurlyeq k[d(T x, y)+d(T y, x)]=(k(x+y), k(x+y)),
$$

where $k=\left(k_{1}, k_{2}\right)$.

Thus $\frac{1}{4} y^{2}+\frac{1}{2} y<k_{i}(x+y)=k_{i} x+k_{i} y<k_{i}\left(\frac{1}{4} y^{2}+\frac{1}{2} y\right)+k_{i} y$, where $i \in\{1,2\}$,

so $\left(1-k_{i}\right)\left(\frac{1}{4} y^{2}+\frac{1}{2} y\right)<k_{i} y$ and $\left(\frac{1}{4} y^{2}+\frac{1}{2} y\right)<\frac{k_{i}}{1-k_{i}} y<y$.

We see that $\frac{k_{i}}{1-k_{i}}<1$ only for $k_{i}>\frac{1}{2}$, so $k>\left(\frac{1}{2}, \frac{1}{2}\right)$ and $\rho(k)>\frac{1}{2}$. Thus, our result is more general than the result of [7] and [8].

Remark 2.2 In Theorem 2.1 we prove the existence but not the uniqueness of fixed point for pseudo contractive mappings. If we take $\rho(h)<\frac{1}{2}$, we prove the uniqueness of fixed point as in following theorem. 
Theorem 2.2. Let $(X, d)$ be a complete cone metric space over a Banach algebra $\mathrm{A}, P$ be a solid cone in A. Suppose that the mapping $T: X \rightarrow X$ is quasi contractive, so it satisfies:

$$
d(T x, T y) \preccurlyeq k \max \{d(x, y), d(T x, x), d(T y, y), h[d(T x, y)+d(x, T y)]\}
$$

where $h k=k h, \rho(k)<1$ and $\rho(h)<\frac{1}{2}$. Then $T$ has a unique fixed point in $X$.

Proof. The existence can be proved as in Theorem 2.1 because $\rho(h)<\frac{1}{2}$ and $\rho(k h)<\rho(k) \rho(h)<1$.

Now we prove the uniqueness.

Suppose that exist $y^{*}$ another fixed point of $T$, then

$$
\begin{gathered}
d\left(x^{*}, y^{*}\right)=d\left(T x^{*}, T y^{*}\right) \preccurlyeq \operatorname{kmax}\left\{d\left(x^{*}, y^{*}\right), d\left(T x^{*}, x^{*}\right), d\left(T y^{*}, y^{*}\right), h\left[d\left(T x^{*}, y^{*}\right)+d\left(T y^{*}, x^{*}\right)\right]\right\} \\
=k \max \left\{d\left(x^{*}, y^{*}\right), 2 h d\left(x^{*}, y^{*}\right)\right\}
\end{gathered}
$$

Case 1. If $\max \left\{d\left(x^{*}, y^{*}\right), 2 h d\left(x^{*}, y^{*}\right)\right\}=d\left(x^{*}, y^{*}\right)$, we have $d\left(x^{*}, y^{*}\right) \preccurlyeq k d\left(x^{*}, y^{*}\right)$.

Thus $(e-k) d\left(x^{*}, y^{*}\right) \preccurlyeq \theta$, by multiplying both sides with $(e-k)^{-1}$ we have $d\left(x^{*}, y^{*}\right)=0$ so $x^{*}=y^{*}$

Case 2. If $\max \left\{d\left(x^{*}, y^{*}\right), 2 h d\left(x^{*}, y^{*}\right)\right\}=2 h d\left(x^{*}, y^{*}\right)$, using the same technique as [8], we have:

$$
d\left(x^{*}, y^{*}\right) \preccurlyeq 2 k h d\left(x^{*}, y^{*}\right) \preccurlyeq(2 k h)^{n} d\left(x^{*}, y^{*}\right), n \geq 1 \text {. }
$$

Since $\rho(h k)<\frac{1}{2},\left\|(2 k h)^{n}\right\| \rightarrow 0$, so $\left\{(2 k h)^{n} d\left(x^{*}, y^{*}\right)\right\}$ is $c-$ sequence and $d\left(x^{*}, y^{*}\right) \ll c$, thus $d\left(x^{*}, y^{*}\right)=0, x^{*}=y^{*}$

This result is true for quasi contractive mappings in cone metric space. So we have:

Corollary 2.1. Let $(X, d)$ be a complete cone metric space, $P$ be a solid cone in A. Suppose that the mapping $T: X \rightarrow X$ is quasi contractive, so it satisfies:

$$
d(T x, T y) \preccurlyeq k \max \{d(x, y), d(T x, x), d(T y, y), h[d(T x, y)+d(x, T y)]\}
$$

where $k<1$ and $h<\frac{1}{2}$. Then $T$ has a unique fixed point in $X$.

\section{References}

[1] Banach. S, (1922). Sur les operations dans ensembles abstraits et leur applications aux equations integrals. Fund. Math, 3, 133-181.

[2] Ciric, L. B. (1974). Ageneralization of Banach's contraction principle. Proc. Amer. Math. Soc., 45, 267-273.

[3] Rhoades, B. E. (1997). A comparison of various definitions of contractive mappings. Transactions of the American Mathematical Society, 226, 257-290

[4] Huang, L.-G., \& Zhang. X. (2007). Cone metric spaces and fixed point theorems of contractive mappings. J. Math. Anal. Appl, 332, 1468-1476

[5] Karapinar, E. (2009). Fixed point theorems in cone Banach spaces. Fixed Point Theory Appl., 9.

[6] Rezapour, S., \& Hamlbarani, R. (2008). Some notes on the paper "Cone metric spaces and fixed point theorems of contractive mappings". J. Math. Anal. Appl., 347, 719-724.

[7] Liu, H., \& Xu, S. (2013). Cone metric spaces with Banach algebras and fixed point theorems of generalized Lipschitz mappings. Fixed Point Theory Appl., 320.

[8] Xu, S., \& Radenović, S. (2014). Fixed point theorems of generalized Lipschitz mappings on cone metric spaces over Banach algebras without assumption of normality. Fixed Point Theory Appl., 102

[9] Rudin, W. (1991). Functional Analysis, 245-248. New York: McGraw-Hill.

[10] Kadelburg, Z., \& Radenović, S. (2013). A note on various types of cones and fixed point results in cone 
metric spaces. Asian J. Math. Appl.

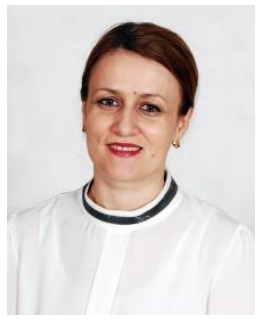

Eriola Sila was born in Lushnja, Albania. She obtained both her master and Phd degrees from the University of Tirana, in 2003 and 2015 respectively. In 2003, she became a lecturer at Department of Mathematics, Faculty of Natural Sciences, University of Tirana, Albania. Her major research interest is "Fixed point theory and its applications in geometry.

Dr. Sila is author and coauthor of several papers, which she has presented at numerous conferences at national and international level. Recently, she has published the book "Transformimet Gjeometrike" (2016) (Geometric Transformations).

Dr. Sila is a member of advisory and assessment board for bachelor and master of science in mathematics studies. She is also a member (an organizer) of Kangoroo Sans Frontiere Olimpiad in Albania.

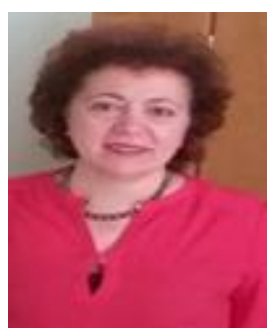

Elida Hoxha was born in Shkodra, Albania. She completed her M. Sc, in mathematics from Tirana University in 1984 and completed her Ph. D. from Tirana University in 1997.

She is a popular professor in undergraduate and post graduate level. Her subjects of teaching are mathematical analysis, topology and functional analysis.

Besides teaching she is actively engaged in research field. Her research fields of fixed point theory, fuzzy sets and fuzzy mappings, topology.

She has a teaching expirience of more than 28 years. Presently, she is working as professor in Department of Mathematics, Faculty of Natural Science, University of Tirana, Albania.

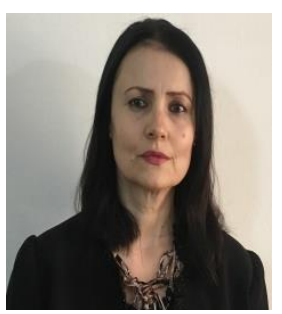

Silvana Liftaj was born in Lushnja, Albania. She obtained both her master and Phd degrees from the University of Tirana, in 2008 and 2015 respectively. In 2010, she became a lecturer at Department of Mathematics and Informatics, Faculty of Information Technology, University "Aleksander Moisiu", Durres, Albania. Her major research interest is fixed point theory in various spaces.

Dr. Liftaj is author and coauthor of several papers, which she has presented at numerous conferences at national and international level.

Dr. Sila is a member of advisory and assessment board for bachelor and master of science in mathematics studies. 